Methods Between June 2010 and 2012, self-collected (SVS) and clinician-collected vaginal (CVS) swabs were obtained from 79 young reproductive age women attending the Prerana Reproductive Health Clinic in Mysore, India. The study was explained to each participant and a brochure illustrating how to collect vaginal swabs was handed to them. The Gram-stained smears and saline wet mounts prepared from the SVS and CVS were examined by a trained microbiologist and the clinician. Vaginal $\mathrm{pH}$ was recorded for each swab. Kappa coefficient was used to quantify agreement between the two sets of results. Results When compared with the CVS, the ability of the selfobtained Gram stain to diagnose bacterial vaginosis had a sensitivity of $100 \%$, specificity of $98 \%$, positive predictive value of $100 \%$ and negative predictive value of $100 \%$. Only one pair was discordant in the results where the SVS showed the BV status as negative while the CVS found it to be intermediate stage BV. There was substantial agreement $(\mathrm{kappa}=0.97)$ between the two collection methods in the ability to determine the grade of vaginal flora.

Conclusion As compared with clinician collected vaginal smears, self-collected smears have substantial agreement in the diagnosis of bacterial vaginosis. With adequate education and instructions using simple visual illustrations, it is possible to have women sample and self collect vaginal swabs for diagnosis of lower genital tract infections.

\section{P3.074 MEN'S PREFERENCES FOR STD SCREENING PROGRAMS: INITIAL PREVALENCE OF STD IN THE STUDY POPULATION}

doi:10.1136/sextrans-2013-051184.0534

'B Van Der Pol, 'A Davis, 'A D Smith, ${ }^{2} \mathrm{~J}$ A Williams, ${ }^{2} \mathrm{G}$ Zimet. 'Indiana University School of Public Health, Bloomington, IN, United States; Indiana University School of Medicine, Indianapolis, IN, United States

Background STD control efforts in the US and western Europe have had less than desirable impact, in part due to an inability to reach populations of men at risk for these diseases. We are currently conducting a study of programme options, including self-collection of specimens and community based access to test kits, that would increase men's utilisation of screening services.

Methods Using peer-incentivized referral, a type of snowball sampling, beginning with men attending an STD clinic in the US, we are interviewing men in the community to determine the optimal combination of programme features that would encourage asymptomatic STD screening. To demonstrate the relevance of these men's opinions in terms of reaching a high prevalence group, they were tested for STDs at the time of their interview. Urine samples were collected and rectal sampling was offered to all men. STD testing was performed using nucleic acid amplification testing.

Results To date, 25 men from the community have been enrolled and interviewed. $40 \%$ of participants were black, $40 \%$ were white, and $20 \%$ were Hispanic. The median age of participants is 24 . Urine samples were available for testing from all 25 men and rectal samples were available from 5 men. $4(16 \%)$ men tested positive for a STD: $2(8 \%)$ men tested positive for chlamydia and $2(8 \%)$ men tested positive for trichomonas. No gonococcal infections were detected. None of the rectal samples had a positive result.

Conclusion While these results represent pilot data, the study is ongoing and given the nature of snowball sampling, the sample size will expand rapidly. Early prevalence rates are higher than have been reported in nationally representative surveys in the US. This suggests that we are recruiting from a population of interest to gather opinions about preferred screening options.

\section{P3.075 TRENDS IN EPIDEMIOLOGY AND MANAGEMENT OF REPORTABLE SEXUALLY TRANSMITTED INFECTIONS (STIS) IN POLAND}

doi:10.1136/sextrans-2013-051184.0535
A Serwin, 'M Koper, ${ }^{2} \mathrm{M}$ Unemo, ${ }^{3} \mathrm{M}$ Domeika, Eastern European Network for SexualReproductive Health. 'Department of Dermatology and Venereology, Medical University of Bialystok, Bialystok, Poland; ${ }^{2}$ WHO Collaborating Centre for Gonorrhoea and other STIs, Örebro, Sweden; ${ }^{3}$ Department of Control and Prevention of Communicable Diseases, Uppsala County Council, Uppsala, Sweden

Background In Poland, among STIs syphilis and gonorrhoea are reportable since 1947, non-gonococcal genitourinary infections (NGU) - 1980, HIV infections - 1985, genital herpes, and anogenital warts (AGWs) - 1990

Methods . Analysis of data on the incidence and management of gonorrhoea, syphilis, NGU, genital herpes, AGWs and HIV infections/AIDS cases available from reports of the National Institute of Public Health and the former Institute of Venereology, Poland.

Results Gonorrhoea. The average incidence rate was 1.24 per 100000 inhabitants during 2000-2011. Culture of Neisseria gonorrhoeae remains the mainstay of diagnosis and ceftriaxone is the recommended first-line treatment. Syphilis. The mean incidence rate from 2000 to 2011 was 1.91 per 100000 inhabitants. The number of serological tests for syphilis (STS) performed gradually diminished from 1200000 in 2000 to less than 100000 in 2011. In the same period 637 cases of syphilis in pregnancy or at delivery and 119 cases of congenital syphilis were diagnosed. The number of notified NGU cases decreased from 1981 in 2000 to 484 in 2011, that of genital herpes declined from 418 to 173 in respective years, and that of reported AGWs fluctuated (range from 400 to 1400 cases per year). Since the start of testing in 1985, 16314 cases of HIV infection and 2848 AIDS cases have been diagnosed until 2013. About $20 \%$ of all HIV infections were related to risky heterosexual intercourse. The incidence of notified HIV infection in Poland is increasing, while the incidence of the remaining reportable STIs is declining.

Conclusions The increasing HIV incidence and decreasing number of STS along with presence of congenital syphilis cases are of major concern. The reporting system of STIs in Poland is suboptimal.

\section{P3.076 CANADIAN TRENDS IN SEXUALLY TRANSMITTED INFECTIONS, 2002 TO 2011}

doi:10.1136/sextrans-2013-051184.0536

S L Totten, A Kemp, J Njihia, C Archibald. Public Health Agency of Canada, Ottawa, ON, Canada

Background In Canada, there are three nationally notifiable sexually transmitted infections (STI): chlamydia, gonorrhoea, and infectious syphilis (includes primary, secondary, and early latent infections). Surveillance of these infections involves local, provin$\mathrm{cial} /$ territorial and federal levels of government. This analysis examines trends in the rates of reported cases of these STIs from 2002 to 2011.

Methods Reportable STI data are compiled at the national level to examine trends over time by age, sex, and geographical distribution of cases across the country. Data for 2011 are preliminary; therefore, sex-specific rates are not available at this time.

Results Preliminary reported rates of chlamydia and gonorrhoea in 2011 were 290.2 and 33.1 per 100,000, respectively. Rates increased $62 \%$ for chlamydia and $41 \%$ for gonorrhoea from 2002 . Youth and younger adults have the highest rates of these infections, particularly in females. However, in older adults $(40+$ for chlamydia and $25+$ for gonorrhoea), males have higher rates.

The rate of reported cases of infectious syphilis in 2011 was 5.1 per 100,000 (an increase of $240 \%$ from 2002). Increases have been driven almost entirely by cases reported among men; in 2010, it was reported in nearly 10 times as many males as females. Males aged 20 to 59 have high rates of reported infectious syphilis.

Conclusion While the implementation of nucleic acid amplification testing beginning in 1997 likely contributed to the initial rise in 